\title{
L'impératif territorial (Partie 2)
}

\section{Denis Retaillé}

\section{(2) OpenEdition \\ Journals}

\section{Édition électronique}

URL : http://journals.openedition.org/conflits/636

DOI : $10.4000 /$ conflits.636

ISSN : $1777-5345$

Éditeur :

CCLS - Centre d'études sur les conflits lilberté et sécurité, L'Harmattan

\section{Édition imprimée}

Date de publication : 15 mai 1996

ISSN : 1157-996X

\section{Référence électronique}

Denis Retaillé, «L'impératif territorial (Partie 2) », Cultures \& Conflits [En ligne], 21-22 I printemps-été 1996, mis en ligne le 28 février 2005, consulté le 30 mars 2021. URL : http://journals.openedition.org/ conflits/636 ; DOI : https://doi.org/10.4000/conflits.636

Ce document a été généré automatiquement le 30 mars 2021.

Creative Commons License 


\title{
L'impératif territorial (Partie 2)
}

\author{
Denis Retaillé
}

1 La volonté de monde Simpliste depuis la dénonciation du " fardeau de l'homme blanc ", la critique du tableau européo-centré et le différentialisme poussé jusqu'à l'essentialisme présentent l'universalisation et la mondialisation comme aboutissements de l'impérialisme et déviation inacceptable de la diversité et de l'autonomie des civilisations au profit d'une seule. La dénonciation porte sur des acteurs dominants qui sont de plus en plus des institutions froides, voire des mécanismes insaisissables comme ceux qui se développent dans la sphère financière. Je ne vois pas beaucoup d'efficacité à cette analyse de la situation globale, sinon dans l'ordre de la mobilisation pour la contre-mondialisation. Alors réémerge l'impératif territorial, paradoxalement, dans le camp de ceux qui dénoncent l'aboutissement de la territorialisation du monde sur le mode européen : ethnarques, Prophètes, visionnaires politiques. Pour ou contre le Monde, le Territoire est là, en amont de l'Histoire, comme une entrée; en aval, comme une sortie ou un refuge! Si les formes spatiales des groupes humains ne sont pas déjà dessinées dans la nature mais résultent de l'organisation des sociétés et des contacts qu'elles entretiennent, résultent même de la projection dans la réalité de l'imagination géographique qui se développe à partir d'indices superficiels (un nombre, un produit, un paysage etc.), alors il faut examiner l'intentionnalité de tous ceux qui initient la mise en forme pour tenter de fixer le passage de la géographie spontanée à la volonté géographique ou géographie " politique ". Elle exprime l'exercice d'un pouvoir, et, pour réussir, doit s'appuyer sur un minimum de légitimité. L'invention du monde résulte d'une volonté de monde ou, plus exactement, de volontés coalisées ou affrontées, ajoutées ou retranchées, combinées en savoir et pouvoir. Revenons à l'individu comme unité incontestable du genre humain, libre ou pas. Il ne doit sa survie consciente qu'à l'entretien de relations avec des semblables. Mais dans le même temps, aucun de ses congénères ne peut résider au même emplacement que lui. Un groupe humain local, parfaitement primitif, parfaitement isolé et fermé, assurant sa simple survie c'est-à-dire sa reproduction, porte donc sur un " territoire " qui est à la fois ressource et somme des distances séparant chacun des individus de tous les autres. Au degré zéro, la société est un groupe humain habitant un territoire exploité selon une répartition des tâches établie 
par lieu, et soudé par une coordination qui ressemble déjà à l'exercice de la fonction politique. Le territoire-ressource constitue une première enveloppe d'identité spatiale, que l'individu en exploite directement et physiquement des produits ou, plus souvent maintenant, qu'il y trouve tous les individus (et les fonctions) dont il a besoin pour survivre dans sa dimension triviale. Le territoire-ressource, c'est l'espace des nécessités vitales $^{1}$. A l'échelle de l'individu toujours, la dimension de l'interconnaissance qui comprend le contact physique, l'affect et la familiarité, fixe la territorialité limitée à un horizon incompressible mais susceptible d'évolution selon la relation sociale, la capacité technique, les pas de temps envisagés. La Territorialité c'est surtout l'expérience de l'unité. Le territoire est fait d'ancres et de bornes partagées par les membres du groupe, qui le caractérisent et le différencient de ce qui constitue l'extérieur, l'espace de l'aventure. Le sentiment ou la perception instituent des seuils, des passages d'un lieu à un autre, d'un territoire à l'autre. Matérielle ou intellectuelle, la marque de la différence qui sépare les lieux est un étage supérieur de l'identité géographique. Au-dessus de l'appartenance territoriale qui tient dans une forme d'immédiateté, la non appartenance ne peut s'exprimer qu'à travers la connaissance relative. A la familiarité s'ajoute, en opposition, l'altérité construite en correspondance, terme à terme. Le paysage, la langue, la forme du contrôle social... Tout peut participer à la reconnaissance de la différence qui distancie les lieux ou mieux les " territoires ". J'appelle " Géographicité " cette expérience de la différence par la distance ${ }^{2}$. Territorialité et Géographicité ne se construisent pas tout à fait au hasard. L'intelligence de la surface terrestre n'est pas non plus uniforme; elle ne se moule pas dans une seule raison et ne se limite pas à une valeur physique des milieux selon un classement unique, celui de la productivité végétale par exemple. La compréhension complète des écarts n'est possible qu'à la condition d'ajouter à ce couple de l'identité géographique un niveau plus élevé de l'abstraction, la géométrie mentale par laquelle chacun dispose les lieux à la surface de la terre. Pour des raisons de commodité et surtout de rapidité, examinons les opposés classiques: nomades et sédentaires. Ils n'envisagent pas l'espace de la même manière. Le territoire nomade est constitué d'un ensemble de lieux éloignés qui ne valent que pour autant qu'ils sont réunis dans une circulation plus ou moins régulière. La territorialité porte sur la disponibilité rythmée en eau et en herbe, hiérarchisée par la priorité d'accès. La distance topographique importe relativement peu (encore qu'il faille nuancer selon les capacités de circulation). En revanche, les distances écologiques sont très prégnantes. Elles se marquent par la qualité de l'herbe, le volume d'eau, les conditions sanitaires animales, la saisonnalité. Il s'y ajoute des distances sociales selon l'ouverture ou la fermeture des passages, la liberté de l'eau, la domination ou la soumission. Tout cela dessine une géographicité qui est simplement l'intelligence des lieux. Mais la forme de cette géographicité n'est composée que de points et de lignes, disons d'itinéraires hiérarchisés intégrés ou tronçonnés. La qualité de l'itinéraire et la valeur d'une direction comptent seules. En face, - c'est préjuger de l'existence d'une seule forme spatiale - ou bien au-dessous - c'est préjuger de la saillance d'une forme sur l'autre -, les sédentaires paysans définissent le territoire par le terroir d'abord ou l'ensemble des terroirs complémentaires qui constituent le pays. L'exploitation y est continue dans le temps (investissement foncier) et dans l'espace borné de la " propriété ". La surface est le module spatial de base, sa dimension fait sa valeur. Nul besoin de nous y arrêter plus longuement. Au contact nomade/sédentaire un problème se pose donc. Avons-nous affaire à deux espaces spécialisés par les modes d'exploitation du milieu et les sociétés, 
limités par un seuil " physique " de pluviométrie par exemple, ou avons-nous affaire à deux formes spatiales superposées (des itinéraires et des surfaces) dont la saillance de l'une n'est que le signe d'une supériorité politique. J'appelle " Spatialité " cette géométrie par laquelle se pense l'espace terrestre et dont nous verrons l'importance lorsqu'il faudra s'habituer à ne pas ramener tous les territoires à une seule métrique, dite topographique, celle du terroir justement, la surface clairement délimitée. Ce qui apparaît dans notre analyse réaliste classique comme conflit de compétence " territoriale " peut être beaucoup plus complexe lorsque des spatialités différentes sont en jeu. Il nous faudra apprendre à manipuler ensemble diverses métriques de l'espace des sociétés. Sociétés agraires ou sociétés industrielles, marché local ou transaction globale, les conditions générales de l'identité géographique se modifient considérablement. Quelles formes sont saillantes sur les autres, quelles formes sont englobantes ? N'est-ce pas affaire de choix et finalement de politique, expression de la volonté de monde qui s'exprime chez des acteurs concurrents. Que devient l'impératif territorial dans tout cela? N'est-il pas un vieil héritage de civilisation agraire dépassée, héritage de la cohésion assurée par la coalition des soldats et des paysans qui traitent la terre comme une ressource primaire? Ou bien, dans une variété de métriques qui reste à reconnaître, n'est-il pas la condition de l'organisation sociétale. C'est une première série de questions. Nous croyons découvrir aujourd'hui, dans le monde très contemporain, une ambivalence indépassable devant laquelle l'analyse se trouve désarmée, et qui constitue le motif de notre rassemblement. L'espace des sociétés est fait de réseaux et de territoires. Tant que l'État a été l'individu-acteur privilégié par la science politique, le territoire fut incontestable. Tellement qu'il n'a pas été pensé autrement qu'en termes géopolitiques : à toi, à moi, grand, petit, riche, pauvre, allongé, trapu, massif, délié, au centre, à la périphérie, continental, maritime, continu, discontinu etc. Tout s'est passé comme si nous n'avions pu prendre en compte, mesurer, éventuellement déplacer, que des stocks, des masses, des solides bien définis, sertis... mais pas les mouvements flous, aléatoires qui sont ceux des fluides qu'on ne peut mesurer qu'en flux ${ }^{3}$. Pour aider à l'analyse positive, ce qui n'est que flux de pensée, de vie, d'initiative a été coagulé en solides: des peuples, des territoires, des richesses. Alors l'impératif territorial est aussi vrai pour le pouvoir que pour le savoir : c'est l'établissement d'un déterminisme simple qui permet d'établir l'information et de prévoir l'action. De cette situation, le passage au réseau marquerait l'éparpillement de l'information et l'éclatement du pouvoir de contrôle, un monde sans ordre d'où s'effacerait la fonction politique au profit de la concurrence des règles: le marché contre l'identité, l'information contre le mythe. Est-ce si simple? La saillance du réseau sur le territoire est-elle une parfaite nouveauté ? (C'est vrai que nous sommes habitués à l'englobement du réseau par le territoire). L'impératif territorial disparait-il avec le primat du territoire? Quelle forme prend-il ? Ce sont les nouvelles questions ou les questions oubliées que doit traiter la géographie du Politique. Deuxième série. Début de réponse. La sorte de géographie " spontanée ", fondamentale, structurale et inconsciente qui pourrait s'écrire selon les considérations précédentes, s'en tiendrait aux représentations sociales de l'espace terrestre et à leur mise en oeuvre, sans du tout s'inquiéter de l'intentionnalité des acteurs. Or, c'est la volonté de monde exprimée par les acteurs dominants et entraînants que veut cerner la géographie du politique, en poussant l'analyse jusqu'à la responsabilité, c'est-à-dire au rebord de la science, juste avant l'action. Dans le monde dominé par l'État ou dans toute forme exclusive et exhaustive de partage, la géographie n'a guère été sollicitée que pour évaluer 
l'allocation des ressources localisées. Il allait de soi que tous les fonctionnements sociaux étaient réglés sur le même principe: unité interne, altérité absolue de l'extérieur. Il allait de soi aussi que la définition de la société pouvait s'atteindre par sa délimitation, deux opérations confondues déjà observées dans l'invention du monde. Il reste à identifier le médium qui produit l'unité interne et s'interpose dans toute relation externe pour établir le lieu d'exercice de la fonction politique. La géographie qui s'occupe de cette manière est " politique " par ses tableaux et géopolitique par ses principes de compréhension. Les ressources étant localisées, une action volontaire d'allocation et de transformation de l'allocation ne peut être qu'une action de prédation, interne ou externe. A l'intérieur, les règles économiques et sociales orientent la distribution des richesses; à l'extérieur, la diplomatie et au besoin la guerre servent à défendre les intérêts " communs " ou " solidaires " de la société interne. Ce couple géographie politique - géopolitique, a connu son développement maximal avec le jeu de puissances. Le monde y est pensé en surfaces, selon un assemblage tout à fait particulier des métriques sociétales, topographique au dedans, topologique au dehors. Topologique d'abord. Il s'agit d'une manière de mesurer les passages brusques d'un lieu à l'autre, d'un niveau hiérarchique à l'autre, d'un territoire à l'autre. La frontière est un seuil privilégié d'observation, c'est le seul qui aille jusqu'à l'exclusive. C'est dedans ou dehors mais ni entre les deux ni ailleurs. Topographique ensuite. Cette manière de mesurer s'applique à suivre la continuité physique, la chaîne de proximité. Une unité topographique, c'est une surface devenue lieu, une étendue dont on a annulé toute distance pour privilégier l'appartenance solidaire mais qui n'en conserve pas moins une dimension. Exclusivité topologique, exhaustivité topographique par la fusion de toutes les fonctions sociales dans l'État ou la Nation, une portion découpée de l'humanité et de la terre en même temps est un pays. Quand il devient beaucoup trop coûteux d'assurer sa puissance en poursuivant la tendance autarcique en même temps que l'activité de prédation (pour simplifier), mieux vaut échanger en se plaçant le mieux possible dans la circulation des biens et des idées, en proposant aux autres ses propres symboles ou en digérant les symboles extérieurs etc. De ce moment, les entités géographiques ne se définissent plus comme des surfaces globales que sont les pays mais comme des réseaux dont les lieux sont les connexions. C'est le lieu qui compte, participant plus ou moins aux flux et dont la position est clairement identifiable. La prédation n'est plus l'activité dominante, celle qui donne le pouvoir ou oriente son exercice. La participation lui a succédé. Alors la géographie passe de la mesure des ressources localisées à celle des interactions, beaucoup plus intéressante en terme politique. En effet, la géopolitique soumet entièrement l'action à la stratégie militaire et diplomatique (géostratégie) ou administrative (aménagement du territoire et éventuellement découpage électoral). La géographie des interactions, quant à elle, doit envisager tous les acteurs, y compris ceux de la société civile, dont les stratégies peuvent être concurrentes ou convergentes en termes de lieux, toujours plus complexes. Les acteurs géopolitiques restent à leur besoin de puissance interne et externe, traitent le territoire selon cet impératif et simplifient les enjeux par l'opposition du à moi et du à toi. Les acteurs en réseau, eux, militent pour la position, pensent en même temps le lieu et le monde et cherchent à minimiser le coût de la surface qui constitue une distance supplémentaire et un poids. La métrique permettant de mesurer l'activité sociale des acteurs en réseau (appartenance interne et relations avec l'extérieur) est de type topologique sur les deux faces (position dans le réseau, connexions ou non au réseau). L'impératif territorial semble disparu, lui qui est 
approximativement lié à une métrique interne de type topographique. Ce n'est pas encore si simple. Retenons pour le moment que toutes les activités sociales ne s'inscrivent pas de la même manière dans l'espace, non seulement du fait de dispositions à caractère " culturel " mais du fait de leur nature même. C'est comme d'affirmer que l'espace de production n'a ni la même structure ni la même forme ni la même extension que l'espace de circulation. Pourtant les deux se rencontrent! Même l'économie réelle et la sphère financière conservent quelques attaches et peuvent embrayer, y compris dans des réactions erratiques. N'en va-t-il pas de même dans le champ du politique? Non seulement les espaces se rencontrent comme se rencontrent les différents secteurs de la vie sociale, mais peut-être appartient-il justement au politique d'encore organiser l'interaction, pour le profit de tous. Question de responsabilité qui s'accompagne d'un prolongement. Jusqu'où? C'est alors un problème de définition de la société. Nous y revoilà. Un groupe humain, un territoire, l'exploitation des ressources, le partage des tâches et la coordination: une société globale, quels que soient son étendue et son nombre, se définit par l'interaction des différentes fonctions productrices de société. Aux deux extrémités de la liste des composantes sociales se situent les limites de définition. Ou bien le groupe humain existe en soi avec tous ses attributs; ou bien il est le résultat de l'entrée en interaction de diverses fonctions sociales et l'on voit mal comment la fonction politique pourrait ne pas être la courroie de transmission de l'événement déclencheur: une guerre, une conquête de peuplement, une révolution ${ }^{4}$. Or, pour obtenir l'interaction maximum entre les différentes fonctions sociales, il est nécessaire de les contenir dans les mêmes limites. La production d'une société est territorialisante en ce sens. Le politique qui porte la responsabilité des choix ne peut se satisfaire d'une dilution des conséquences d'orientation dans un horizon illimité au risque de perdre toute légitimité et éventuellement tout sens. Dans la production sociétale par le politique se trouvent l'exhaustivité tendancielle et la limite. L'exclusivité n'est qu'une conséquence. Ce mixte sociétal qui soude société civile et société politique en une totalité indissociable, est viable dans des conditions de dimension qui sont très variées. Selon la base économique et la volonté de monde, tout peut changer, la dimension topographique, la métrique sociétale, l'échelle du fonctionnement englobant. Agriculture, mine et puissance doivent disposer d'un territoire vaste et exclusif ; échange et richesse se contentent de petites dimensions à la condition d'une forte densité (toutes les fonctions sociétales dans un réduit éventuellement) et d'une connexion bien établie avec une grande variété de lieux; industrie et développement situent les besoins géographiques d'une société à mi-chemin de la réserve d'espace et du lieu en s'orientant vers le contrôle des ressources sans obligatoirement passer par l'appropriation. Alors les positions ne sont pas les mêmes: fermeture et accumulation dans le premier cas, nous pensons là aux empires " militaires "; ouverture et participation dans le second, les cités marchandes s'y retrouvent en position intermédiaire, l'État balance entre la territorialité topographique et l'organisation de l'échange lorsqu'il ne fait pas la guerre. Dans le continuum de ces situations, les choix apparaissent comme des intersections du politique et du géographique, en imprimant à la société une métrique et une échelle d'existence et de fonctionnement qui définissent son identité. L'invention du monde continue donc à la mesure de la volonté qu'expriment les acteurs dominants. Il est certes fini le monde, mais il change sans cesse de forme autant que ses éléments changent de position. Il est fini, mais la complexité gagne, avec le croisement des phénomènes contradictoires de mondialisation de certains secteurs des sociétés, 
matériels surtout, et de contre-mondialisation d'autres secteurs, culturels au sens identitaire. Mais il fut aussi des époques où les idées ont plus circulé que les produits. Les allures locales qui ont ainsi accumulé des héritages d'origine et d'époque variées, en atteignent l'infinie diversité. Alors, outre le découpage simplificateur en surfaces contiguës, des plans d'organisation peuvent être aisément compris et déchiffrés : le monde des identités culturelles séparées, le système interétatique, le marché mondial hiérarchisé, l'information mondiale. Nous y retrouvons territoires et réseaux. Les interactions entre les plans sont même identifiables : insertion, inclusion, intégration, différenciation, fragmentation, enclavement ${ }^{5}$. Mais à partir du moment où une fonctionnalité sociétale se place à l'échelle du monde et dans la métrique des réseaux, une conséquence doit être tirée. L'impératif territorial étant coextensif de la fonction la plus englobante, l'organisation de la complémentarité et de la concurrence, le respect ou l'anéantissement des différences, tout cela se joue désormais à l'échelle du monde et concerne chaque lieu. L'impératif territorial a été technique et cartographique pendant la période de découverte. Le savoir a guidé le pouvoir et l'a parfois installé. Difficile de dire que le politique, qui est tout de même encore le lieu du pouvoir, doit, maintenant que le monde est plein, guider le savoir ou en susciter de nouveaux développements (c'est la déviation de la géopolitique). Pour moi, c'est juste à côté du Politique que s'établit la relation pouvoir/savoir et la définition d'une science (géographique) du Politique. Le problème nouveau que nous pensons ne pas savoir/pouvoir résoudre de manière réaliste, la concomitance de la mondialisation des phénomènes et de la fragmentation des identités, propulse la pensée de l'universel vers l'éthique et la délimitation du niveau local vers l'identité. Cela réduit le champ d'investigation d'une nouvelle géographie. Entre l'éthique et l'identité se développe l'action politique qui est gestion et orientation des distances séparant les deux bornes. Voilà un champ géographique. Entre le monde et le lieu, tous les niveaux intermédiaires ne sont que constructions et étapes stratégiques selon le nouvel impératif territorial qui engage la responsabilité des acteurs dans les deux directions. Voilà un champ politique. Jusqu'alors, la coalescence des sociétés avait été simplement traitée par la contiguïté parfaite et la première formule de l'impératif territorial. Maintenant c'est la coalescence des plans de la société qu'il faut inventer. Quelque chose qui ressemble au fédéralisme horizontal c'est-à-dire à l'assemblage de sociétalités inachevées ou incomplètes en des lieux imparfaits, un peu fuyants, et de dimensions très variables ${ }^{6}$. Quelle justice et quelle légitimité dans ce monde apparemment mal ajusté ? Les rebondissements ne cessent jamais. La question est même mal posée. La justice et la légitimité peuvent-elles être le ciment de ces morceaux de mondes établis sur des plans disjoints ? Je n'entrevois qu'à peine les implications d'une telle réflexion malgré Rawls ${ }^{7}$. Depuis que se pratiquent l'aménagement du territoire ou les politiques d'aide au développement, les géographes évoquent la justice spatiale ${ }^{8}$.Elle peut prendre deux allures assez différentes qui ne sont pas sans rappeler l'opposition des métriques et l'enjeu fondamental de l'impératif territorial. Ou bien, cas le plus fréquent et aussi le plus trivial, on pense à l'égalisation des stocks par portion d'espace (des " régions " de même étendue, ou de même population, ou de même puissance, disposant des mêmes structures etc.) la " départementalisation " pour tout dire ; ou bien la complémentarité et la concurrence sont privilégiées en préservant la différence, mais dans l'équivalence des situations (égale faculté de choix, égale faculté d'ouverture ou de fermeture etc.) en plaçant tout lieu dans la position de participer au mouvement d'ensemble. Le territoire ou le réseau se retrouvent, le saupoudrage ou l'entraînement, la campagne ou la ville : 
les aimants du balancier des politiques géographiques sont partout les mêmes. Et à l'échelle globale deux utopies, c'est le lieu de le dire, peuvent se développer. La " départementalisation " du monde c'est la transformation des territoires hérités ou inventés en circonscriptions de gestion des stocks disponibles ou alloués. Les " départements " (territoires) peuvent être regroupés en " régions " mondiales pour tirer profit de proximités ou dépasser des seuils d'économie d'échelle et d'efficacité. C'est la voie ouverte par le fédéralisme vertical qui reconnaît à chaque palier une compétence totale dans la limite des règles reconnues par tous. Mais la concentration du lieu de décision et l'élargissement de ses compétences peuvent transformer le monde " départementalisé " en empire-monde. La " polarisation " du monde c'est, à l'inverse, si l'on peut dire, l'attention portée aux lieux (à l'échelle locale de l'apparition de la société globale) et à leur interaction. Mais l'émergence sociétale ne se fonde pas partout sur les mêmes principes; un même individu peut, en outre, appartenir à plusieurs " sociétés " et plusieurs " lieux ", certains définis par la culture, d'autres par le fonctionnement économique ou politique. Alors les métriques comme les échelles s'embrouillent. C'est le domaine de la multiple appartenance qui ne trouve de solution que dans la réduction asymptotique des distances - toujours l'impératif territorial - par la ville globale désormais ${ }^{10}$. Au total, trois opérations élémentaires cernent l'activité géographique, dont la hiérarchie varie avec la volonté de monde. Localiser, découper, relier : Localiser, c'est identifier un lieu en lui donnant son nom (un nom), sa position et sa dimension. Découper, c'est identifier des lieux en traçant leurs limites, en leur donnant une substance et une place en situation. Relier, c'est identifier les lieux en repérant une position, une substance et une échelle de fonctionnement. La variation des opérations réside dans le changement d'ordre et dans une légère réorientation des concepts qui manifestent une mutation méthodologique sur le versant scientifique et une mutation politique sur le plan de l'action. Prenons quelques exemples: localiser Palestine ou Israël, choisir le nom entraîne une cascade de conséquences qu'on imagine immédiatement tant sur le plan de la position (alliances, développement etc.) que sur celui de la délimitation. Et comment! Découper les territoires des Slaves du Sud : après avoir localisé, c'est-à-dire nommé une substance ethnique ici, trancher et répartir ne peut être que parfaitement arbitraire (le passage des limites dépend de l'échelle d'observation et des références). Relier. L'opération est simple dans les cas de continuité-contiguïté. C'est rassembler. Elle l'est moins lorsque le phénomène est " discontigu ". L'initiative entreprise pour les Amériques, dans le premier cas, ressemble à une intégration en gradient d'États déjà alliés en plusieurs groupes régionaux plus ou moins serrés. Ce n'est que le projet d'un emboîtement fédéral vertical. L'Union européenne ressortit à ce type. Dans le second cas, je crois pouvoir trouver la mamlaka (forme spatiale de l'Umma), aussi bien qu'IBM ou la diaspora chinoise. Ce sont des réseaux pour lesquels l'impératif territorial joue à la plus vaste échelle sans nécessité de continuité topographique. La connexion suffit. Alors qu'est-ce qu'un chinois musulman employé de Barings-Singapour? Dans quel ordre prononcer l'identité ? Qui en détermine la pertinence? La Science politique a privilégié l'État dans son observation des relations internationales pour, dit-on, atteindre le statut de " Science " positive, rendre possible des mesures, aller et venir entre théorie et vérification empirique $^{11}$. Elle se confinait dans un espace solide pour acquérir une sécurité de mécanicien. Une grande attention portée à ce que les acteurs disent d'eux-mêmes, ce qu'ils sont, ce qu'ils font, comment ils sont et comment ils font, ouvre un nouveau champ scientifique, considérablement élargi en termes spatiaux. Cette libération 
géographique nous affranchit de l'unique référence à l'espace matériel. C'est de la plus grande conséquence si l'on songe à la transposition dans l'action. Examiner la conscience et l'intentionnalité des acteurs ne suffit plus. Il faut aller jusqu'à comprendre (et forger) l'organisation du champ collectif, qui est le monde, par la substitution de l'acte à l'esprit, de la réalité du mécanisme subjectif à l'idéal de la conscience ${ }^{12}$, atteindre la condition de la légitimité : l'entre-soi ; transformer l'attribut du territoire : ressource puis empire, enfin lieu de légitimité. Poursuivre l'invention du monde.

Revenir à la partie précédente

\section{NOTES}

1. J. Brunhes, La géographie humaine, édition abrégée, Paris, PUF, 1942.

2. D. Retaillé, Représentations et enjeux de l'espace au Sahel, Vol. IV, ronéo, Université de Rouen, 1993.

3. M. Serres, Atlas, Paris, Julliard, 1994.

4. Collectif, La société civile, CURAPP, Paris, PUF, 1986.

5. M.F. Durand, J. Lévy, D. Retaillé, Le monde, espaces et systèmes, Paris, 2ème éd.

Dalloz, Presses de la Fondation Nationale de Sciences Politiques, 1993.

6. J. Lévy, L'espace légitime, Paris, Presses de la Fondation Nationale des Sciences Politiques, 1994.

7. J. Rawls, Théorie de la justice, trad. française, Paris, Le Seuil, 1987.

8. A. Reynaud, Société, espace et justice, Paris, PUF, 1981

9. M. Guillaume, Une fin de siècle épidémique, Arles, Actes Sud, 1992.

10. Collectif, Les relations internationales à l'épreuve des sciences politiques, Mélanges Marcel Merle, Paris, Economica, 1993.

11. Collectif, Les relations internationales à l'épreuve des sciences politiques, Mélanges Marcel Merle, Paris, Economica, 1993.

12. M. Gauchet, Le désenchantement du monde, Paris, Gallimard, 1985.

\section{INDEX}

Mots-clés : géographie, sciences politiques, territoire(s) et territorialité, souveraineté 